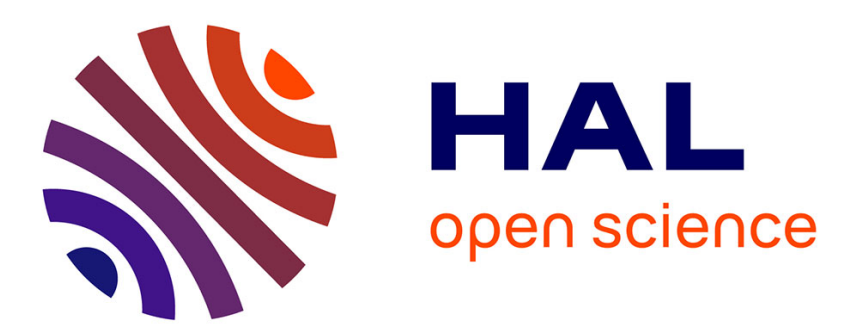

\title{
Surface plasmons in the Young slit doublet experiment
}

Sylvain Ravets, Jean-Claude Rodier, Buntha Ea-Kim, Jean-Paul Hugonin, Lionel Jacubowiez, Philippe Lalanne

\section{To cite this version:}

Sylvain Ravets, Jean-Claude Rodier, Buntha Ea-Kim, Jean-Paul Hugonin, Lionel Jacubowiez, et al.. Surface plasmons in the Young slit doublet experiment. Journal of the Optical Society of America B, 2009, 26 (12), pp.B28-B33. hal-00566689

\section{HAL Id: hal-00566689 \\ https://hal-iogs.archives-ouvertes.fr/hal-00566689}

Submitted on 5 Apr 2012

HAL is a multi-disciplinary open access archive for the deposit and dissemination of scientific research documents, whether they are published or not. The documents may come from teaching and research institutions in France or abroad, or from public or private research centers.
L'archive ouverte pluridisciplinaire HAL, est destinée au dépôt et à la diffusion de documents scientifiques de niveau recherche, publiés ou non, émanant des établissements d'enseignement et de recherche français ou étrangers, des laboratoires publics ou privés. 


\title{
Surface plasmons in the Young slit doublet experiment
}

\author{
S. Ravets, J. C. Rodier, B. Ea Kim, J. P. Hugonin, L. Jacubowiez, and P. Lalanne* \\ Laboratoire Charles Fabry de l'Institut d'Optique, CNRS, Université Paris-Sud Campus Polytechnique, \\ RD 128, 91127 Palaiseau, France \\ *Corresponding author: philippe.lalanne@institutoptique.fr
}

Received April 24, 2009; revised June 12, 2009; accepted July 1, 2009;

posted July 23, 2009 (Doc. ID 110551); published August 18, 2009

\begin{abstract}
We present an in-depth analysis of Young's slit experiment when solely a single slit of the doublet is illuminated by a focused laser beam at visible and thermal infrared wavelengths. In line with the recent results obtained by Kuzmin et al.. [Opt. Lett. 32, 445 (2007)], even if only a single slit is illuminated by a focused beam, we show that the far-field pattern exhibits sinusoidal Young's fringes. Thanks to fully vectorial electromagnetic computations and to a simple model, we provide a comprehensive discussion of the nature of surface waves at work in the experiment. (c) 2009 Optical Society of America
\end{abstract}

OCIS codes: $260.1960,240.6680$.

\section{INTRODUCTION}

Young's slit doublet experiment is emblematic in optics. It is generally thought to have been first performed by the English scientist Thomas Young during the year 1801 in an attempt to resolve the question of whether light was composed of particles or rather consisted of waves. The interference patterns observed in the experiment seemed to discredit the corpuscular theory, and the wave theory of light remained well accepted until the early 20 th century, when evidence began to accumulate that seemed instead to confirm the particle theory of light. The double-slit experiment and its variations then played an essential role in the discussion of the foundations of quantum mechanics [1]. Later, the experiment was repeated with electrons detected with a closely spaced grid of electron detectors [2] or with atoms [3]. More recently, the initial experiment with photons has been revisited with a slit doublet patterned with modern nanolithography tools and illuminated by a large spectrum source. A surprising modulation of the intensity transmitted by the doublet as a function of the wavelength has been observed [4-9]. This modulation has been interpreted as a direct signature of the embodiment of surface plasmon polaritons (SPPs).

In 1957, Ritchie theoretically predicted that when a metal is in the form of a thin foil, charge oscillations of free metal electron gas take place at the top and bottom interfaces of the metal film. This collective oscillation has been observed experimentally and is presently known as an SPP [10]. Theoretically, an SPP simply arises as a purely $2 \mathrm{D}$ mode solution of Maxwell's equations that propagates as a transverse magnetic mode along the metallo-dielectric interface. The mode is bounded (it decays exponentially in the metal and in the dielectric) on the interface and propagates with a complex propagation constant, $k_{\mathrm{SP}}=k_{0}\left(\epsilon_{\mathrm{m}} \epsilon_{\mathrm{d}} /\left(\epsilon_{\mathrm{m}}+\epsilon_{\mathrm{d}}\right)\right)^{1 / 2}, \epsilon_{\mathrm{m}}$ and $\epsilon_{\mathrm{d}}$ being the relative permittivities of the metal and of the dielectric, respectively. Note that at optical frequencies for noble metals, $\epsilon_{\mathrm{m}}$ is complex and $\operatorname{Re}\left(\epsilon_{\mathrm{m}}\right) \ll 0$. Because the SPP is a bounded mode, it cannot be directly excited when a flat interface is illuminated by a plane wave, even at highly oblique incidence. However, SPPs are launched when the translation invariance is broken, for example, by etching a subwavelength indentation in a metal thin film.

With this in mind, we may indeed anticipate that SPPs should be involved in Young's fringe experiment. Their embodiment has been evidenced by direct near-field measurements [11] performed at $\lambda=974 \mathrm{~nm}$, showing that the immediate vicinity of the slit doublet above the sample is composed of a stationary interference pattern. The latter can be understood as mainly resulting from two counterpropagating SPPs that are launched by the slits. Even more surprisingly, Young's fringes have been recently observed with good visibility, even when a single slit of the doublet is illuminated by a focused beam $[12,13]$.

The present work is primarily concerned by this last experiment that we repeat here in the near-infrared domain $(\lambda=810 \mathrm{~nm})$. A priori, the physical interpretation is quite straightforward: the illuminated slit scatters the incident beam and launches an SPP that travels towards the partner slit and is converted there to free-space radiation. Therefore, the propagating SPP allows an additional path for light transmission through the slit doublet.

Section 2 describes the experimental setup and the sample used to observe Young's far-field pattern with good visibility. In Section 3, we quantitatively compare the experimental results with fully vectorial computational results and study the fringe visibility as a function of the slit widths. In contrast with the earlier works in $[12,13]$, we further evidence that the fringe pattern is observed whether the sample is illuminated from the front side (air-gold interface) or from the rear side (glass-gold interface). With a simple model (relying on the reciprocity theorem applied to SPP modes) that is consistent with the experimental and computational results, we explain why the observation of a modulation with good visibility is independent of the illumination side. Section 4 is devoted to 
a more general discussion of the influence of metal conductivity in the experiment. Through computational results we predict that Young's fringe pattern should be observed at longer wavelengths, in the thermal infrared or even in the microwave regimes, without any SPP registering. We therefore refine the simplistic initial picture of a pure SPP description.

The numerical data presented hereafter are all obtained with a fully vectorial frequency-domain modal method relying on Fourier expansion techniques, the aperiodic Fourier modal method (a-FMM) $[14,15]$. For the slit doublet scattering problem considered in this work, the a-FMM provides highly accurate numerical solutions of Maxwell's equations, as evidenced by its successful benchmarking for a related groove slit problem [16]. For the computations, gold is considered as a real metal with finite conductivity, and the gold frequency-dependent permittivity $\epsilon_{\mathrm{m}}\left(\epsilon_{\mathrm{m}}=-27.3+1.9 \mathrm{j}\right.$ at $\left.\lambda=810 \mathrm{~nm}\right)$ is taken from [17].

\section{EXPERIMENTAL RESULTS}

The experimental arrangement is shown in Fig. 1(a). The incident light is issuing from a SuperK broadband supercontinuum white light source [18]. The light is further filtered by a $10 \mathrm{~nm}$ bandwidth filter $(\mathrm{F})$ around a central wavelength $\lambda=810 \mathrm{~nm}$. The exit face of the monomode photonic-crystal fiber is imaged onto the sample through two microscope objectives (L1) and (L2). A simple CCD camera (not shown in Fig. 1) is used to accurately align the illuminating laser spot on one slit of the doublet. A half-wave plate is used to control the polarization of the incident light, which can be either parallel (TE) or perpendicular (TM) to the long axis of the slits. The far-field pattern is recorded by a CMOS camera that is approximately situated at a $5 \mathrm{~mm}$ distance from the rear side of the sample.

(a)

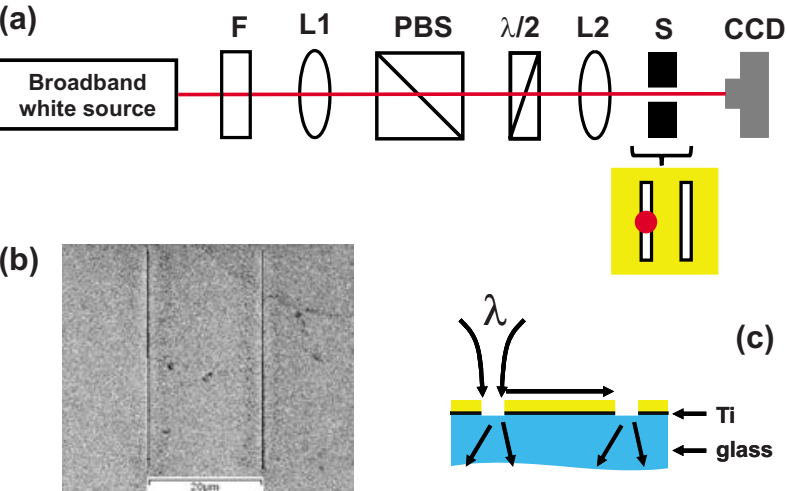

Fig. 1. (Color online) (a) Experimental setup for measuring transmission through the slit doublet when solely a single slit is illuminated by a focused Gaussian beam emitted from a photonic-crystal fiber. F, L1 and L2, PBS, S, CCD denote the $10 \mathrm{~nm}$-large spectral filter, objective lenses (N.A. $=0.18$ for L1 and 0.35 for L2), polarizing beam splitter, sample, and CCD camera, respectively. (b) Scanning electron microscope picture of a doublet. (c) Pathway of light and SPP, when the sample is normally illuminated from the front side (air cladding). The nominal thicknesses of the gold and titanium layers are $200 \mathrm{~nm}$ and $5 \mathrm{~nm}$.
The subwavelength structures are etched by electronbeam lithography (nB3 apparatus from NanoBeam Ltd. operating at $80 \mathrm{kV}$ with $3 \mathrm{~nm}$ beam size) followed by ion beam etching (using a 3 inch diameter ion gun source of Veeco at $200 \mathrm{eV}$ ) in a $200 \mathrm{~nm}$ thick gold layer evaporated onto a $0.5 \mathrm{~mm}$ thick fused-silica substrate. Figure 1(b) shows a scanning electron microscope (SEM) picture of the slit doublet obtained at $20 \mathrm{kV}$. The sample consists of a 2D array of slit doublets with different center-to-center separation distances $-d=20,40$, and $50 \mu \mathrm{m}$-and with different slit widths varying from 200 to $1200 \mathrm{~nm}$ by a $50 \mathrm{~nm}$ step. Every slit is $20 \mu \mathrm{m}$ long. The following results are obtained for doublets with $d=20 \mu \mathrm{m}$, but similar results have been obtained for other separation distances. Note that a $5 \mathrm{~nm}$ thick Titanium film lies between the glass substrate and the gold film, as shown in Fig. 1(c). The thin layer serves two purposes. Primarily, it acts as an adhesion layer for the gold film deposition. It additionally prevents any electromagnetic interaction between the two slits at the rear substrate interface, because the characteristic damping length of the SPP supported by the glass/Ti/gold interface is only a few wavelengths for a $5 \mathrm{~nm}$ Ti thickness. Consequently, of all the interfaces that we probe in the experiment, only the air/gold one supports SPPs propagating over distances comparable to the slit separation distances. This important remark will be used hereafter to interpret the experimental data.

Figure 2 summarizes the main results obtained when illuminating a single slit with a focused beam. Let us first consider an illumination from the front side of the sample [light incident from air, see Fig. 2(a)]. For TM polarization, we observe fringes with good visibility as a function of the diffracted angle $\theta$. The fringe pattern visibility, defined as $C=\left(\mathrm{I}_{\mathrm{M}}-\mathrm{I}_{\mathrm{m}}\right) /\left(\mathrm{I}_{\mathrm{M}}+\mathrm{I}_{\mathrm{m}}\right)$ where $\mathrm{I}_{\mathrm{M}}$ and $\mathrm{I}_{\mathrm{m}}$ stand for the maximum and minimum intensities, is $\approx 0.20$ for $\theta$ $=0$. When instead a TE polarization beam is used to illuminate the sample, the detected pattern shows no modulation [see the right-hand inset in Fig. 2(a)]. The fact that

(a)

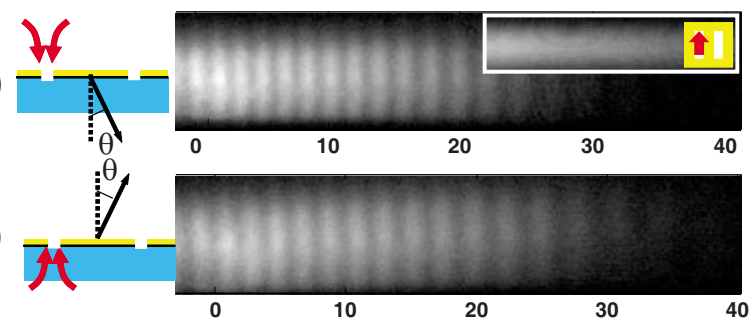

(c)

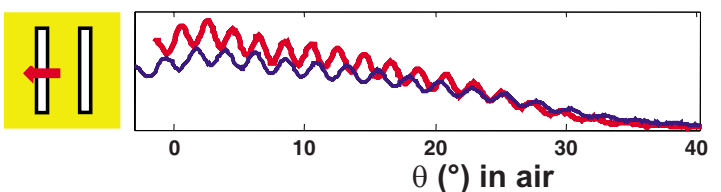

Fig. 2. (Color online) Interference patterns recorded on the CCD camera for a doublet with slit widths of $300 \mathrm{~nm}$. (a) TM polarization for a Gaussian beam incident from air. Inset, TE polarization for a Gaussian beam incident from air. (b) TM polarization for a Gaussian beam incident from the glass substrate. (c) Cross sections of the recorded patterns shown in (a) and (b). Thick curve, incidence from air. Thin curve, incidence from glass. The fringe periodicities of the curves are slightly different and are slightly shifted because the relative position between the camera and the sample is not kept constant when rotating the sample between the two experiments. 
we observe interference fringes for the TM-polarized illumination case demonstrates that the fields emerging from the two slits must be at least partially mutually coherent [13]. In the geometry of our sample, the SPP travels from one slit to the other with little loss, the slit separation $(20 \mu \mathrm{m})$ being smaller than the SPP decay length $\left(1 / \operatorname{Im}\left(k_{\mathrm{SP}}\right) \approx 90 \mu \mathrm{m}\right.$ at $\left.\lambda=810 \mathrm{~nm}\right)$. At the second slit, the SPP is partially converted back into a propagating light field. The fringe visibility, $C \approx 0.20$, reflects the unbalance of the intensities of the two fields emerging from the two slits.

Figure 2(b) is obtained for TM polarization and for an incident illumination impinging from the rear side of the sample (light incident from glass). Again the far-field pattern is composed of fringes that are very similar to those obtained when illuminating the front side. This is exemplified by Fig. 2(c) that compares the fringe cross sections obtained along the interference patterns.

\section{COMPARISON WITH THEORETICAL RESULTS}

To get more insight into the experimental results, we further resort to computation. Figure 3 shows the magnetic near field that we have calculated with the a-FMM for TM polarization when the right-hand slit is illuminated by a focused Gaussian beam. Figures 3(a) and 3(b), respectively, correspond to an illumination from the front and rear sides of the sample. The insets show enlarged views in the vicinity of the left-hand slit, the one that is not illuminated. It is amazing to observe that the magnetic

(a)
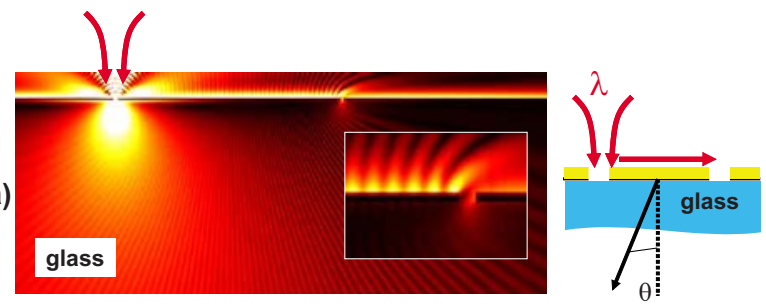

(b)
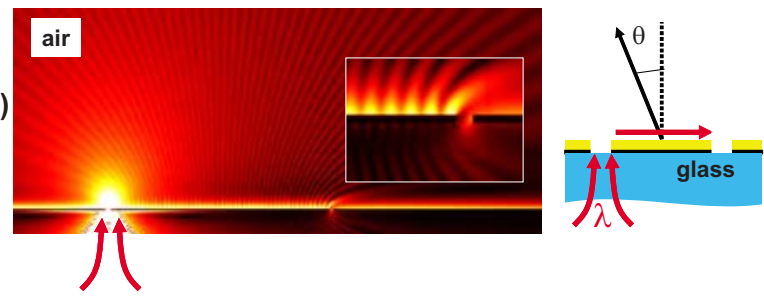

Fig. 3. (Color online) Calculated near-field pattern (magneticfield modulus) for TM polarization. (a) Illumination from air. (b) Illumination from the glass substrate. The insets show an enlarged view in the vicinity of the slit that is not illuminated. Note that the SPP of the air-gold interface is much stronger than that of the glass-gold interface. The calculation is performed for a wavelength $\lambda=810 \mathrm{~nm}$, with gold relative permittivity $\epsilon_{\mathrm{m}}=$ $-27.3+1.9 \mathrm{j}$. The waist of the normally incident Gaussian beam is $2 \lambda$. Slit widths are $300 \mathrm{~nm}$, slit separation distance is $20 \mu \mathrm{m}$, gold thickness is $200 \mathrm{~nm}$, and adlayer Ti-thickness is $5 \mathrm{~nm}(n$ $=2.9+3.3 \mathrm{j}$ ). Be aware that the two images are shown with a highly nonlinear scale in order to evidence the existence of the near-field fringe between the slits. The fringes are mainly due to the interference between the right-traveling SPP (launched from the left-hand slit) and the left-traveling SPP that is backreflected from the right-hand slit (see inset). field distributions of the two figures are very similar. They are both consistent with the previous interpretation based on SPPs launched at the air/gold interface.

From the near-field computational results, we then perform a plane-wave decomposition and calculate the far-field radiation diagram as a function of the diffracted angle $\theta$. The dashed curve in Fig. 4 shows the calculated data. The latter are obtained for a Gaussian beam, illuminating the sample from the front side (airgold interface), focused on a single slit, and for monochromatic light $(\lambda=810 \mathrm{~nm})$. We also calculate data for polychromatic light over a spectral linewidth, $\Delta \lambda=10 \mathrm{~nm}$ (solid thin curve). They exhibit a slightly reduced contrast as predicted by the degree of coherence, $\gamma$ $=\operatorname{sinc}\left[\Delta \lambda / \lambda\left(k_{\mathrm{SP}}-k_{0} \sin (\theta)\right) d / 2\right]$, which is approximately equal to 0.85 for $\theta=0$.

We have repeated the experiments for several doublets, varying the slit widths $w$. The fringe visibilities measured for $\theta=0$ are shown in Fig. 5. The computed visibilities, shown with solid curves, are slightly larger than the experimental values. Amazingly, we note that for $\theta=0$, the visibility does not depend on the illuminated sample side.

To further analyze all of the data, we develop a simple SPP model. Figure 6(a) shows the elementary SPP scattering coefficients at a metallic interface perforated by an isolated subwavelength slit when the slit is illuminated by an incident plane wave (left) or by its fundamental guided mode (right). Because the slit is symmetric, only three complex coefficients $\alpha, \beta(\theta)$, and $t(\theta)$ have to be considered. In Figure 6(b), we show a related scattering problem where the slit is illuminated by an SPP. This defines two new scattering coefficients, $\alpha^{\prime}$ and $\beta^{\prime}(\theta)$. We refer to the reciprocity theorem (time-reversal arguments do not apply in the present case because of absorption) [19] to guarantee that to $\alpha=\alpha^{\prime}$ and $\beta(\theta)=\beta^{\prime}(\theta)$; see details in [20] for SPPs.

Figure 6(c) shows the situation where the sample is illuminated at normal incidence from the front (air) side. The incident light excites the fundamental mode of the

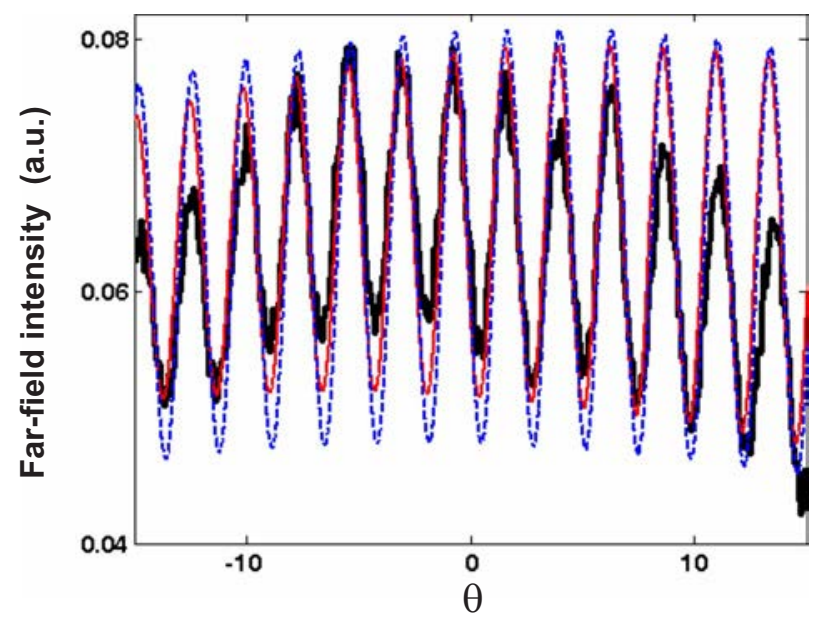

Fig. 4. (Color online) Comparison between measured and calculated far-field fringes. Solid thick curve, experimental data. Solid thin curve, calculated data for polychromatic light over spectral linewidth $\Delta \lambda=10 \mathrm{~nm}$. Dashed curve, calculated data for monochromatic light $(\lambda=810 \mathrm{~nm})$. The results are obtained for a Gaussian beam incident from the substrate. 


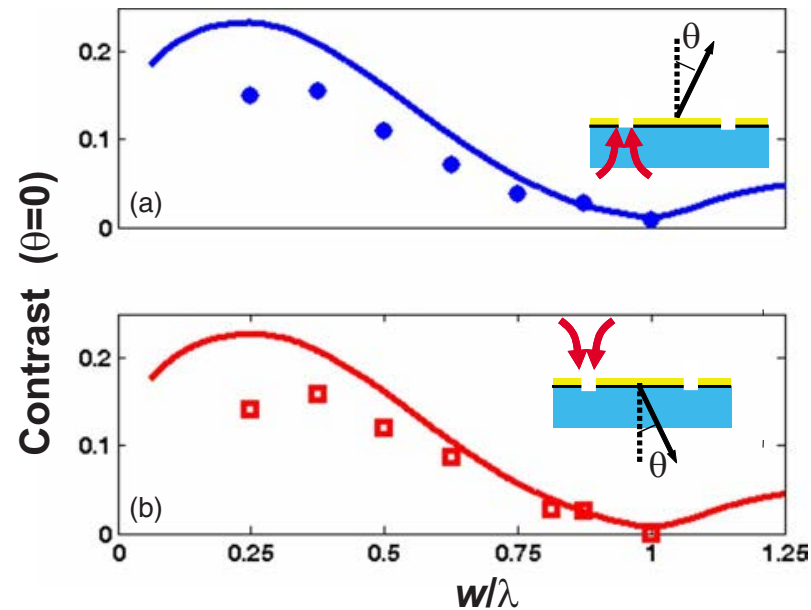

Fig. 5. (Color online) Fringe visibilities as a function of the normalized slit widths. (a) Illumination from the glass substrate. (b) Illumination from air. The solid curves represent computed data obtained for an illumination with a $10 \mathrm{~nm}$ spectral bandwidth. The dot and square marks are visibilities deduced from the experimental data for the central fringes close to $\theta=0$.

left-hand illuminated slit with a complex coefficient $t_{a}(0)$, where the subscript "a" (or"g") is related to the transmission at the air/gold (or glass/gold) interface. It additionally launches an SPP that propagates to the right with amplitude $\beta(0)$. The SPP further scatters at the nearby slit and excites the slit fundamental mode with amplitude $\alpha^{\prime} \beta(0) \exp \left(\mathrm{i} k_{\mathrm{SP}} d\right)$. The fringe pattern results from the interference of two waves scattered by the two slits. In the

(a)
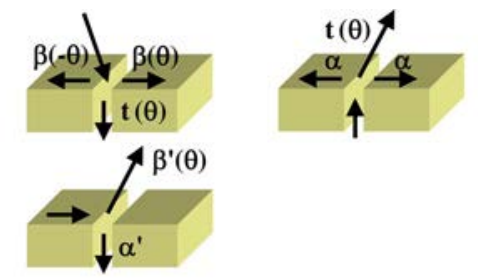

(b)

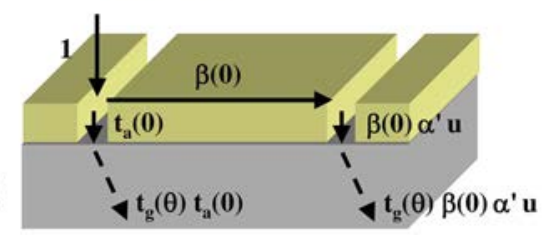

(d)

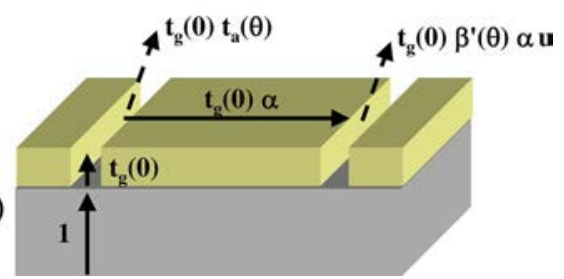

Fig. 6. (Color online) Simple model for the pathways of light and SPP in the slit doublet experiment. (a) SPP generation at a metallic interface perforated by a single slit under illumination by an plane wave with incidence angle $\theta$ (left) of by the fundamental mode of the slit (right). Only two scattering coefficients $a$ and $\beta(\theta)$ need to be defined because of the symmetry with respect to the slit axis. (b) Related scattering coefficients. Under adequate normalization, the reciprocity theorem guaranties that $\alpha=\alpha^{\prime}$ and $\beta(\theta)=\beta^{\prime}(\theta)$. (c) Illumination from the front (air) side. (d) Illumination from the rear (glass) side. direction defined by angle $\theta$, the scattered amplitudes are proportional to $\mathrm{t}_{\mathrm{a}}(0) \mathrm{t}_{\mathrm{g}}(\theta)$ and $\alpha^{\prime} \beta(0) \mathrm{t}_{\mathrm{g}}(\theta) \exp \left(\mathrm{i} k_{\mathrm{SP}} d\right)$. Obviously, the contrast is independent of $\theta$, and the intensity of the far-field fringe pattern is proportional to

$$
\begin{aligned}
\mathrm{I}(\theta)< & \left|\mathrm{t}_{\mathrm{g}}(\theta)\right|^{2}(1 \\
& +\frac{2\left|\mathrm{t}_{\mathrm{a}}(0) \alpha^{\prime} \beta(0)\right| \exp \left(-\operatorname{Im}\left(\mathrm{k}_{\mathrm{SP}} \mathrm{d}\right)\right)}{\left|\mathrm{t}_{\mathrm{a}}(0)\right|^{2}+\left|\alpha^{\prime} \beta(0)\right|^{2} \exp \left(-2 \operatorname{Im}\left(\mathrm{k}_{\mathrm{SP}}\right) \mathrm{d}\right)} \cos \left(\operatorname{Re}\left(\mathrm{k}_{\mathrm{SP}}\right) \mathrm{d}\right. \\
& \left.\left.+\mathrm{f}-\mathrm{k}_{0} \mathrm{~d} \sin (\theta)\right)\right)
\end{aligned}
$$

where $\varphi$ is the total phase resulting from the SPP coupling factors. Considering that $\left|\alpha \beta^{\prime}(0)\right|^{2} \ll\left|\mathrm{t}_{\mathrm{a}}(0)\right|^{2}$, the visibility is equal to

$$
\mathrm{C}_{\mathrm{air}}=\left|\frac{2 \alpha^{\prime} \beta(0)}{\mathrm{t}_{\mathrm{a}}(0)}\right| \exp \left(-\operatorname{Im}\left(\mathrm{k}_{\mathrm{SP}} \mathrm{d}\right)\right) .
$$

We now illuminate the sample at normal incidence from the rear (glass) side [Fig. 6(d)]. The scattered amplitudes become $\mathrm{t}_{\mathrm{g}}(0) \mathrm{t}_{\mathrm{a}}(\theta)$ and $\mathrm{t}_{\mathrm{g}}(0) \alpha \beta^{\prime}(\theta) \exp \left(\mathrm{ik}_{\mathrm{SP}} d\right)$, and

$$
\mathrm{C}_{\text {glass }}=\left|\frac{2 \alpha \beta^{\prime}(\theta)}{\mathrm{t}_{\mathrm{a}}(\theta)}\right| \exp \left(-\operatorname{Im}\left(\mathrm{k}_{\mathrm{SP}} \mathrm{d}\right)\right)
$$

In contrast to the previous case, the visibility now depends on $\theta$. However for $\theta=0, C_{\text {glass }}$ and $C_{\text {air }}$ are equal since reciprocity guaranties that $\alpha \beta^{\prime}(0)=\alpha^{\prime} \beta(0)$. Although a deviation exists between the experimental and computational data in Fig. 5, it is clear that the measured visibilities $C_{\text {glass }}$ and $C_{\text {air }}$ are very similar. Thus, the experiment can be considered as direct proof of the reciprocity theorem applied to SPPs. Note, however, that only the equality of the modulus of the scattering coefficients is tested, not that of the complex scattering coefficients themselves.

This simple modal analysis that basically relies on reciprocity and symmetry arguments is valid as long as the energy transfer from the rear to the front sides of the slits is governed by the fundamental $\mathrm{TEM}_{0}$ slit mode [21]. In general, this transfer can be also provided by other propagative (if $w>\lambda / 2$ ) or evanescent (at small film thicknesses) modes. In the present experiment, the gold thickness is rather small, and $C_{\text {glass }}$ and $C_{\text {air }}$ nearly exhibit the same variation with angle $\theta$. However, other computational results (not reported here for the sake of compactness) have confirmed that the single-mode picture is valid for narrow and deep slits. Moreover, the model is also consistent with earlier theoretical results [19] predicting that the modulus of the $\alpha$ and $\beta$ coefficients reach their maximum values for slit widths that are approximately equal to a quarter of the wavelength $(w \sim \lambda / 4)$ and are almost null for $w \sim \lambda$. Finally let us note that the oscillating sinclike variation of the visibility in Fig. 5 is compatible with recent near-field measurements performed on single slits [22]. 


\section{EFFECT OF THE METAL CONDUCTIVITY}

So far we have restricted the discussion to near-infraredwavelength illuminations $(\lambda=810 \mathrm{~nm})$. It is important to apprehend how the SPP picture evolves as one increases the wavelength by scaling all the geometrical parameters accordingly. The answer deserves some attention and has been tackled only recently in the literature [23].

One has first to realize that as the wavelength increases, the metal permittivity increases too. $\varepsilon_{\mathrm{m}}$ approximately scales as $\lambda^{2}$, according to the Drude model [24]. As the wavelength increases, the SPP mode becomes less and less confined on the interface-it spreads far away in the dielectric half-space and weakly feels the electron gas. Its excitation efficiency, $|\alpha|^{2}$ or $|\beta|^{2}$, vanishes for a tiny subwavelength slit. This is intuitively clear if one considers that spatially extended fields cannot be excited efficiently by subwavelength emitters (the slit aperture). This handwaved argument has been theoretically analyzed, and it has been shown that the excitation efficiency scales as $\left(\varepsilon_{\mathrm{m}}\right)^{-1}[20,25]$. Thus one should expect that the visibilities given by Eqs. (2) and (3) rapidly decrease as the wavelength increases.

Actually this is not the case, and the pure SPP vision has to be revised for full understanding. Figure 7 shows the far-field fringe pattern calculated with the a-FMM when a single slit is illuminated under TM polarization by a $2 \lambda$ waist Gaussian beam. The calculation has been performed for various incident wavelengths ranging from the visible to the thermal infrared. For all cases, reasonably large visibilities $(C=0.15-0.30)$ are obtained. Consistent with earlier studies [26], a fringe pattern with $C$ $\approx 0.1$ is obtained even when assuming that the metal is perfectly conducting (PC). PC metals represent a good ap-

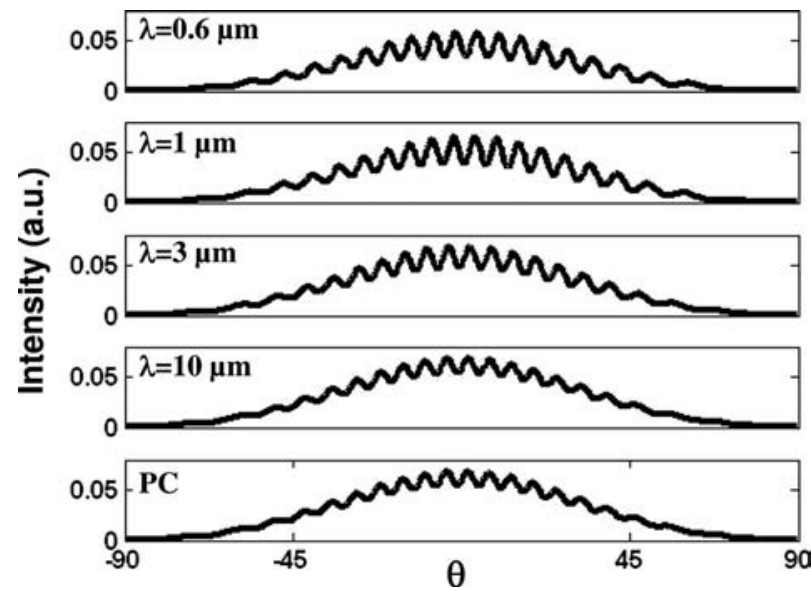

Fig. 7. Calculated far-field fringe pattern of a slit doublet experiment when solely a single slit is illuminated by a focused Gaussian beam. The calculation has been performed with the a-FMM for several wavelengths ranging from the visible (top) to the infrared (bottom) by scaling all the geometrical parameters. Slit widths are $w=0.4 \lambda$, slit separation distance is $d=10 \lambda$, gold thickness is $\lambda / 4$, and adlayer Ti thickness is $\lambda / 160$. Except for the bottom curve where a PC is considered, gold is considered a real metal with finite conductivity and with frequency-dependent permittivity taken from [13]. SPPs are mainly responsible for the fringe existence at $\lambda=0.6$ and $1 \mu \mathrm{m}$, half responsible for the fringes observed at $\lambda=3 \mu \mathrm{m}$, and very weakly involved at $\lambda$ $=10 \mu \mathrm{m}$. They cannot be considered for explaining the fringe pattern predicted for the PC case. proximation in the $\mathrm{THz}$ and microwave domains. As discussed above, SPPs are mainly responsible for the fringe pattern at high energies $(\lambda=0.6-1 \mu \mathrm{m})$, are partly responsible for the fringe pattern obtained at $\lambda=3 \mu \mathrm{m}$, and are almost not involved at longer wavelengths, $\lambda$ $>10 \mu \mathrm{m}[11,23,27]$. This is due to the fact that the field scattered on the metallic surface by a subwavelength slit is not solely composed of an SPP mode, but also encompasses a residual quasi-cylindrical wave (quasi-CW), i.e., an electromagnetic field with radiative and evanescent components that persists along the surface over a few tens of wavelength propagation distances. For noble metals, both waves are equally excited at visible frequencies, but at longer wavelengths the quasi-CW rapidly becomes the preponderant field. Just as SPPs, the quasi-CW participates in the energy transfer between the two slits, and this is a profound reason for the fringe observation at $\lambda$ $=3$ and $10 \mu \mathrm{m}$. The reader may refer to [23] for a quantitative discussion of the respective contribution of the SPP mode and the quasi-CW for related slit-groove scattering geometry.

Finally, let us notice that in Fig. 7 the visibility is maximum for $\lambda=1 \mu \mathrm{m}$. SPPs are excited more efficiently in the visible at $\lambda=0.6 \mu \mathrm{m}$, but this increase is compensated by a larger damping as one approaches the plasma frequency, so the product $\left|\alpha \beta \exp \left(\mathrm{ik}_{\mathrm{SP}} d\right)\right|$ is effectively slightly smaller at $\lambda=0.6 \mu \mathrm{m}$ than at $\lambda=1 \mu \mathrm{m}$.

\section{CONCLUSION}

We have performed a comprehensive study of the enrolment of SPPs in Young's double-slit experiment, especially focusing on situations where a single slit is illuminated by a focused beam. At high energies in the visible range, SPPs have a profound influence on the coherence property of the doublet. At smaller energies, they are no longer involved but the physics remains essentially the same, and the fringe pattern is still observed for TM polarization. In fact, many optical phenomena such as transmissions through arrayed slits or holes, which are observed with metallic nanostructures at visible frequencies, can be reproduced at longer wavelengths by scaling the geometrical parameters.

\section{ACKNOWLEDGMENTS}

The gold deposition was performed at the CTU IEFMINERVE. Electronbeam lithography and Ion beam etching were achieved at the Microtechnology Center of TRT/ IO/X-PALAISEAU.

\section{REFERENCES}

1. N. Bohr, Atomic Physics and Human Knowledge (Wiley, 1958).

2. C. Jönsson, "Electron diffraction at multiple slits," Am. J. Phys. 42, 4-11 (1974).

3. O. Carnal and J. Mlynek, "Young's double-slit experiment with atoms: a simple atom interferometer," Phys. Rev. Lett. 66, 2689-2692 (1991).

4. H. F. Schouten, N. Kuzmin, G. Dubois, T. D. Visser, G. Gbur, P. F. A. Alkemade, H. Blok, G. W. 't Hooft, D. Lenstra, and E. R. Eliel, "Plasmon-assisted two-slit transmission: 
Young's experiment revisited," Phys. Rev. Lett. 94, 053901 (2005).

5. C. H. Gan, G. Gbur, and T. D. Visser, "Surface plasmons modulate the spatial coherence of light in Young's interference experiment," Phys. Rev. Lett. 98, 043908 (2007).

6. D. Pacifici, H. J. Lezec, H. A. Atwater, and J. Weiner, "Quantitative determination of optical transmission through subwavelength slit arrays in $\mathrm{Ag}$ films: role of surface wave interference and local coupling between adjacent slits," Phys. Rev. B 77, 115411 (2008).

7. O. T. A. Janssen, H. P. Urbach, and G. W. 't Hooft, "On the phase of plasmons excited by slits in a metal film," Opt. Express 14, 11823-11832 (2006).

8. H. Shi, X. Luo, and C. Du, "Young's interference of double metallic nanoslit with different widths," Opt. Express 15, 11321-11327 (2007).

9. Y. J. Bao, R. W. Peng, D. J. Shu, M. Wang, X. Lu, J. Shao, W. Lu, and N. B. Ming, "Role of interference between localized and propagating surface waves on the extraordinary optical transmission through a subwavelength-aperture array," Phys. Rev. Lett. 101, 087401 (2008).

10. H. Raether, Surface Plasmons on Smooth and Rough Surfaces and on Gratings (Springer-Verlag, 1988).

11. L. Aigouy, P. Lalanne, J. P. Hugonin, G. Julié, V. Mathet, and M. Mortier, "Near-field analysis of surface waves launched at nano-slit apertures," Phys. Rev. Lett. 98, 153902 (2007).

12. N. Kuzmin, G. W. 't Hooft, E. R. Eliel, G. Gbur, H. F. Schouten, and T. D. Visser, "Enhancement of spatial coherence by surface plasmons," Opt. Lett. 32, 445-447 (2007).

13. N. V. Kuzmin, Interference Effects with Surface Plasmons, PhD dissertation, Leiden University, Leiden, Germany, ISBN/EAN: 978-90-9022593-7 (2008).

14. E. Silberstein, P. Lalanne, J. P. Hugonin, and Q. Cao, "On the use of grating theory in integrated optics," J. Opt. Soc. Am. A 18, 2865-2875 (2001).
15. J. P. Hugonin and P. Lalanne, "Perfectly-matched-layers as nonlinear coordinate transforms: a generalized formalization,” J. Opt. Soc. Am. A 22, 1844-1849 (2005).

16. M. Besbes, J. P. Hugonin, P. Lalanne, S. van Haver, O. T. A Janssen, A. M. Nugrowati, M. Xu, S. F. Pereira, H. P. Urbach, A. S. van de Nes, P. Bienstman, G. Granet, A. Moreau, S. Helfert, M. Sukharev, T. Seideman, F. I. Baida, B. Guizal, and D. Van Labeke, "Numerical analysis of a slit-groove diffraction problem," J. Eur. Opt. Soc. Rapid Publ. 2, 07022 (2007).

17. E. D. Palik, Handbook of Optical Constants of Solids, Part II (Academic Press, 1985).

18. Koheras SuperK compact, http://www.koheras.com.

19. R. J. Potton, "Reciprocity in optics," Rep. Prog. Phys. 67, 717-754 (2004).

20. H. T. Liu, P. Lalanne, X. Yang, and J. P. Hugonin, "Surface plasmon generation by subwavelength isolated objects," IEEE J. Sel. Top. Quantum Electron. 14, 1522-1529 (2008),

21. J. D. Jackson, Classical Electrodynamics, 3rd ed. (Wiley \& Sons, 1998).

22. H. W. Kihm, K. G. Lee, D. S. Kim, J. H. Kang, and Q.-H. Park, "Control of surface plasmon efficiency by slit-width tuning," Appl. Phys. Lett. 92, 051115 (2008).

23. P. Lalanne and J. P. Hugonin, "Interaction between optical nano-objects at metallo-dielectric interfaces," Nat. Phys. 2, 551-556 (2006).

24. M. Born and E. Wolf, Principle of Optics, 6th ed. (Macmillan, 1964), Chap. 13, p. 627.

25. P. Lalanne, J. P. Hugonin, J. C. Rodier, "Approximate model for surface-plasmon generation at slit apertures," J. Opt. Soc. Am. A 23, 1608-1615 (2006).

26. R. Gordon, "Near-field interference in a subwavelength double slit in a perfect conductor," J. Opt. A, Pure Appl. Opt. 8, L1-L3 (2006).

27. K. J. Ahn, K. J. K. G. Lee, H. W. Kihm, M. A. Seo, A. J. L. Adam, P. C. M. Planken, and D. S. Kim, "Optical and terahertz near-field studies of surface plasmons in subwavelength metallic slits," New J. Phys. 10, 105003 (2008). 